\title{
A Design Framework for Smart Ration Shop Using Blockchain and IoT Technologies
}

\author{
D. Malathi ${ }^{1}$, Vijayakumar Ponnusamy ${ }^{2, *}$, S. Saravanan ${ }^{3}$, D. Deepa ${ }^{4}$ and Tariq Ahamed Ahanger \\ ${ }^{1}$ Department of ECE, Kongu Engineering College, Perundurai, 638060, India \\ ${ }^{2}$ ECE Department, SRM IST-KTR, Chennai, 603202, India \\ ${ }^{3}$ Department of CSE, SRM IST, Chennai, 603202, India \\ ${ }^{4}$ Bannari Amman Institute of Technology, Department of ECE, Erode, 638401, India \\ ${ }^{5}$ College of Computer Engineering and Sciences, Prince Sattam Bin Abdulaziz University, KSA, 11942, Saudi Arabia \\ *Corresponding Author: Vijayakumar Ponnusamy. Email: vijayakp@srmist.edu.in \\ Received: 26 July 2021; Accepted: 27 August 2021
}

\begin{abstract}
The Public Distribution System (PDS) distributes the subsidized food and non-food items to poor populations through fair price shops (FPS). The PDS has been criticized for its urban bias and its failure to serve the underprivileged sections of the community effectively. The Current system manual-based data management and ledger management gives rise to much corruption in the process of extricating the poor from those who are less needy. There are chances for the block market because of the current methodology of data management. This article proposes a blockchain technology-based smart ration shop system that uses immutable smart contract-based transactions to overcome the issues. The proposed mechanism will provide more transparency and enable access to all the transactions by everyone. The system utilizes IoT technology for asset tracking while moving the food items from distribution centers to a particular ration shop, which avoids malpractice during the transportation of the goods. Voicebased and text-based user-friendly interfaces in local languages were also developed to provide an effective interaction and improve the usability of the system. The performance of the proposed blockchain network is evaluated and reported.
\end{abstract}

Keywords: IoT; blockchain technology; smart contract; smart ration shop

\section{Introduction}

Under this Digital era, every government office and all the transactions are being digitalized for efficient data management and quick processing. The fair shop for food item distribution suffers a problem of blackmarketing goods. There is no transparency on the allocation of food items for every individual family and the delivery of goods among the ration shops. Notably, illiterate village consumer of fair shops are not able to get information about their utilization of food items and available stock. The transparency issue arises in the ration shop because of the manual management of transactions. Digital India's objective the problems should be handled utilizing the recent latest digital Information Communication Technology (ICT) Technology. Blockchain Technology is one of the newest technology which transparently handles

This work is licensed under a Creative Commons Attribution 4.0 International License, which permits unrestricted use, distribution, and reproduction in any medium, provided the original work is properly cited. 
documents and transactions and provides immutability for documents and translation. This article utilizes blockchain technology to give immutability and transparency for the transaction of and user data management.

Blockchain technology supporting on various services are discussed in the following Tab. 1

Table 1: Different services on blockchain

\begin{tabular}{|c|c|c|}
\hline $\begin{array}{l}\text { S. } \\
\text { No }\end{array}$ & Areas of services & Blockchain implemented \\
\hline 1. & Financial services & $\begin{array}{l}\text { Banking, Stock trading \& hedge funds, crowdfunding, crypto } \\
\text { exchanges, Loans and credit, }\end{array}$ \\
\hline 2. & Travelling and mobility & $\begin{array}{l}\text { Automotive Manufacturing, Car leasing and sales, Ride- } \\
\text { hailing, trucking, }\end{array}$ \\
\hline 3. & Infrastructure & $\begin{array}{l}\text { Industrial IoT, 3D printing, construction, architecture, } \\
\text { building, Real Estate }\end{array}$ \\
\hline 4. & Health care & $\begin{array}{l}\text { Health information exchanges, claims management, Pharma, } \\
\text { research and clinical trials, }\end{array}$ \\
\hline 5. & Public sector & $\begin{array}{l}\text { Government and public records, voting, gun tracking, federal } \\
\text { email, Law enforcement }\end{array}$ \\
\hline 6. & Retail services & $\begin{array}{l}\text { Retail, E-commerce, food and beverages, gift card loyalty } \\
\text { program, }\end{array}$ \\
\hline 7. & Agriculture and mining & $\begin{array}{l}\text { Crops and agriculture, animal husbandry, fishing, logging, } \\
\text { timber, mining }\end{array}$ \\
\hline 8. & $\begin{array}{l}\text { Education, communication and } \\
\text { information services }\end{array}$ & messaging apps, education and academia, publishing, libraries \\
\hline 9. & Entertainment & $\begin{array}{l}\text { Music and entertainment, video streaming, gaming, gambling, } \\
\text { sports management, }\end{array}$ \\
\hline
\end{tabular}

The proposed system has a user-friendly voice interaction system in the local language as a second component for illiterate to make transparent interaction with the system. As a third component, it has an asset tracking mechanism to ensure the delivery of goods without theft on transport. This work is tested in a village near Chennai called Anjur. A brief review of blockchain Technology and its usability in various system designs are reviewed in the following section.

\section{Related Work}

The public distribution system provides food grains to the citizens at reduced rates to ensure affordability and food safety. The scheme is vulnerable to losses of food products due to widespread corruption in the system as well as physical losses that occur in storage and transportation. In this digital era, Blockchain has been an upcoming audit verification tool due to its inbuilt nature, as it is a distributed log for recording transactions. This article highlights that it can serve as a prime utility to substitute the present centralized scheme that is available for the tracking of food products being disseminated by the public distribution system, thereby avoiding any exploitation or corruption [1].

The article discusses the significance of embedding technological advancements to the currently available ration card booklet. The proposed smart card system will include RFID and IoT to avoid 
mismanagement and fraudulent activity being carried out using the current ration distribution system. Using the RFID tag, the user will be authenticated, and an OTP will be required from the user's mobile number to perform a successful transaction. This will update the database for the government's future verification as well as require minimum human efforts [2].

Nowadays, Blockchain technology has been widely used in various sectors, namely, online payments, forensics, supply chain management, and communicating health records. If this can be extended to combine with the Internet of Things (IoT), then it can lead to a certifiable and perceptible network. In this article, the authors have performed an organized survey in regards to the main components of IoT blockchain then investigated a considerable number of well-known blockchain applications. Finally, they have concluded with an active traffic simulation for IoT-Blockchain schemes to exemplify network traffic distribution [3].

In recent times, Electronic goods are being produced, dispersed, and made available around the world. However, maintaining the security and integrity of the supply chain is becoming tough because of the prevalent access of mistrusted hardware, especially forged and duplicate parts. In this article, they have suggested a nonfatal means of assurance in the traceability of electronic products within the supply chain. To implement them, they have created a blockchain-based structure, which assists in tracking and tracing every product when it is being flown in the supply chain. Moreover, the proposed structure is developed on permissioned blockchain, and Hyperledger is utilized for employing the same [4]. The recent technological advancements concerning distributed ledger, the integration of tokenization in sustainable resources portray it as a proof of concept. This article provides documentation of ERC20 standards in which crypto-contracts happen to be an apparent application of WRC tokens. Moreover, this article is a dual attempt to eradicate the divergence among the small, medium, significant endeavors as well as to create a business opportunity with equal prospects for gaining credits in regards to recycled wastewater [5].

In this digital era, food traceability has emerged to be one of the blockchain applications for enhancing the domains of anti-counterfeiting as well as quality assurance. The present food traceability systems do not assure elevated points of system consistency, scalability, and accuracy of information. Furthermore, the process of traceability is lengthy as well as intricate in new supply chain networks. To mitigate these issues, blockchain technology appears to be capable of developing a new ontology for supply chain traceability. In this article, a blockchain combined IoT food traceability scheme is recommended to amalgamate the innovative application of blockchain, IoT machinery as well as fuzzy logic towards a complete traceability shelf life administration system for managing foods that may perish [6].

The article provides information about a case study of a group in the UK's construction site, making efforts to steer a smart contract proposal. During the pre-pilot phase, they have utilized the sensemaking ideology to know about how the actors in supply chain contextualize the prospective impact of blockchain on their trades.it also establishes a novel technology's indication to enhance speculations, prospects, and awareness about the new technology, which in turn molds their following activities during the pilot program. Subsequently, in the pilot, they have made use of the design science technique blended with the business model's theoretical lens to dynamically shape the implementation of blockchain. Finally, this study provides effective insights into how the group members created a blockchain-based supply chain and different issues faced by them [7].

Digital government is defined as the utilization of information technology to assist government activities, connect citizens, and offer government services according to the declaration mentioned in the digital government society's mission statement. In this discussion, the development of the digital government is depicted and conversed, starting from modest early stages to the current intermediary, along with a forecast and a sample of where this extraordinary multidisciplinary area of research and development might proceed within the forthcoming years. In addition, it is asserted that the makeover of 
the trades of government is only the beginning, and it is argued that the primary and irrefutable cases of evolution can be experienced in the next decade [8].

Supply chain management improved through the IoT solutions incorporates unique tags, namely, Radio Frequency Identification and NFC, with products to produce smart tags as well as storing additional details about a product, which in turn assists in tracking them during their lifecycle. The Distributed ledger-Tags solution enters the present situation to provide decentralized, necessary privacy as well as certifiable management of smart tags within a product's lifecycle. This is regarding distributed ledger technology along with ethereum blockchain to arbitrate communications among the stakeholders that happen through a product's exchange process. The article details the DL-Tags solution alongside the analysis of expenditures involved concerning every transaction that is implemented towards the Ethereum blockchain. Furthermore, the recommended solution offers evidence about the product's basis as well as its achievements in the supply chain to avoid tag repetition and exploitation [9].

Nowadays, it is irrefutable to stay away from electronic systems as it is ubiquitous in everyone's personal lives and also in vital infrastructures, namely, power grids, satellite communications, and transportation systems. Due to the swift globalization in semiconductor engineering, it has been hard to maintain the hardware's reliability and security aspects. This paper details the integrity issues connected with the electronics supply chain from a global perspective. To mitigate these issues in the reliability of the supply chain, a blockchain-based credential authority structure has been proposed to administer the details of critical chip, namely, identification of electronic chip, time of the transaction, and chip's ranking. Furthermore, the decentralized aspect of the recommended structure can alleviate the primary concerns within the electronics supply chain: namely, recycling, remarking, replicating, and unwanted production [10].

The primary requirement of the blockchain is to create an effective framework between independent stakeholders in an unexpected distributed environment. A blockchain system is safe and self-governing in regards to chained blocks, peer-to-peer nodes, ledger techniques on an agreeable limit, unidentified accounts, and self-synchronized data ownership. Also, a dedicated credit schema is necessary to create an effective blockchain system even though it offers an efficient infrastructure for data storage and related activities. The requirement, viability, competence, and required paybacks are vital aspects to examine a blockchain initiative. New speculation of the blockchain is in the pipeline, namely, Facebook's The Libra and Central Bank of China's The DCEP [11].

This article looks at the advancements in blockchain technologies that assure to provide novel tools for the administration of private data, thereby offering improved security and privacy for every human being. Despite the argument in this article, the assured advantages are compromised by a considerable move towards the propitiation of private data within these recommendations. Taking into consideration the distinctive ability of blockchain technology applications for imitating and as well as substituting conventional enterprises, this feature may provide some disputes in the form of technology as well as ethical means. To manage these issues and related concerns, this article diagnoses the hidden technoeconomic aspects and normative speculations describing the enhancement of these solutions piling up to a technologically facilitated propertization [12].

In this decade, food safety concerns have begun to crop up within society. To ably diagnose and avoid food safety issues as well to trace the liability, it is irrefutable to create a reliable traceability system. The conventional traceability structures have problems, namely, the invisibility of data, corrupting, and exposing sensitive information. To counteract this, Blockchain can be a potential technology for food safety traceability structure due to its features, namely, irrevocable time vector, smart agreement, and finally, consensus algorithm. This article proposes a system based on Blockchain along with the EPC information services then creates a simulation. There is also a proposal by creating the management 
architecture with on-chain and off-chain data using which traceability system can mitigate the data explosion problem of the Blockchain for IoT [13].

The recent advancements within information technology have paved means for insightful variations in manufacturing around the world. This article spotlights the theoretical and pragmatic challenges and prospects cropping up from the Internet of Things since it offers new means of supply chain operations to a degree with respect to big data and recent changes in the industry's nature. The article recommends various research tracks and according to research systems in the new scenarios. Furthermore, this study focuses on encouraging future research in the domain of big data-driven supply chain management along with IoT, thereby assisting enterprises to enhance their decisions in data-driven operations as well to offer the government an indication to progress and standardize the growth of the big data as well as the IoT industry [14].

The article elucidates approaches for a technique focusing on premature preparation for the incorporation of IoT technologies within logistics practices. Based on the technical aspects of single technologies as well as process requirements, the taxonomy of IoT technologies offers the foundation for a use case explicit estimation of their technical viability. Furthermore, the generated classification is then joined with cost-benefit estimation approaches. The present status of these developments is monitored based on a logistics use case. The article wraps up with the forecast of future developments and details the role of IoT solutions for the conception of Smart Logistics Sectors [15].

This article examines the novel developments in the domain of ubiquitous computing (also known as pervasive) within logistics. In the recent two decades, research concerns in pervasive computing have grown along with the swift development of information and communication technology. Research investigators are focused on developing IoT technologies to integrate smart devices and sensor networks around the world. Recently, pervasive adaptation has been established as a new research challenge to define a system's capacity to identify the scenario and act accordingly independently. For the contemporary ubiquitous computing devices to turn into a completely working state, it requires to counteract the compatibility and security issues, and these problems, along with appropriate solutions, are detailed in this article [16].

In recent times, Blockchain has turned out to be the latest business opportunity, which has grabbed the interest of financial institutions, governments, and various companies. Also, Blockchain has been hovering to be the most exhilarating discovery post the internet as this only lets the world communicate by enabling novel business models according to the online business processes. In contrast, the Blockchain allows us to fix trust concerns more competently through network computing. In this article, they have provided an outline of blockchain research and development along with related articles. Furthermore, it highlights that Bitcoins being the sensation in digital currency, which was introduced by blockchain technology if adopted widely in finance and various other business domains, may progress towards different enhancements along with plenty of opportunities in research fields [17].

A smart contract is defined as an electronic operation practice necessary to digitally aid, authenticate, and implement the conditions in regards to the primary legal contract framed to accomplish the general contractual terms consisting of payments, legal commitments, and fulfillment without any third parties. The existing smart contract projects disclose that most of its applications in the business environment are connected to supply chain management, IoT, and Industry version 4.0 solutions. The article elucidates the research aspect about how and to what level contracting and blockchain techniques can assist the application of collaborative business frameworks for durable entrepreneurial processes within smart supply chains [18].

In recent times, incorporating novel technologies within every management process leads towards an effective advancement, particularly in smart logistics, as it becomes the primary support for the upcoming 
industrial revolution known as Industry 4.0. Blockchain being an integrated technology and a promising one, lets the decentralized and undeniable storage of confirmed data. The intention behind this study is to focus on the different applications of blockchain within smart logistics along with its ability to provide substantial instances of these applications. In addition, this effort was performed by categorizing the applications conforming to four clusters, namely, Transport, Information, Finance, and management, along with sharing the applications of every cluster [19].

With such immense enhancements in living standards, most people have started to concentrate more on food safety and quality in its related products. Hence, consumers feel that it is vital to have a trustworthy system that can trace the source of food products. On the contrary, the present traceability schemes are deficient in transparency; data is mostly stored within the enterprise, then the price of corrupting the data is meager. Moreover, the supply chain nodes are quite simple to elude accountability when there is a rise of product safety or quality concerns within the conventional centralized management techniques, and it is hard to trace the cause of the issues. In this article, a product traceability framework is proposed based on blockchain technology wherein every product's transferring records are continuously tracked through a distributed ledger with the help of smart contracts along with the formation of a chain that can trace back towards the origin of the products. Besides, a system prototype is created based on the testing structure of Trufe [20].

Nowadays, most businesses are focusing on digitizing their processes in accordance with the recent industrial revolution known as Industry 4.0. The focal point of this digital makeover depends primarily on production. Hence the terms, such as "Factory for the Next Generation" or "Smart Factory," are utilized frequently in this theory. Besides, logistics must achieve a better vision to accomplish the necessities of industry 4.0 as durably as possible with respect to the utilization of proper technologies and improving both horizontal and vertical integration within the supply chain partners. In this regard, this article puts forth the gains that can be achieved if logistics processes are digitized as well as evaluates the durability impact if it happens. Furthermore, this investigation is carried out as a case study in various FMCG enterprises and their respective logistics service teams in Turkey based on qualitative techniques and through connected semi-structured meetings [21]. The works [22-24] focuses on only blockchain transaction implementation without testing with actual data of ration card and without providing any user interface for villagers. But our proposed methodology tested on a real ration card dataset and provides a voice-based interface to interact with kiosk systems with native Tamil language interfaces. Usability of the system is very important, especially when it is concerned with uneducated village people; with this concern, an ease of usability test is also carried out under the proposed system.

From the above literature review and work [25], we can conclude that so far, there is no blockchain technology-based ration shop system work that is reported in the literature.

The proposed blockchain Technology-based smart ration shop system development has the following contribution

A design a framework of blockchain Technology-based ration shop is developed

The design framework is deployed in the village and the performance measurements are carried out

Voice-based user interaction framework also developed using Google speech to text and text to speech conversion engine.

The remaining portion of the article is organized as follows: Section 2 provides the framework of the proposed blockchain-based smart ration shop. Section 3 details the implementation details of the proposed framework. Section 4 presents the results obtained on evolution. Section 5 concludes the article with a summary of the work. 


\section{Design Framework of Smart Ration Shop}

The Proposed system utilizes three types of Technology 1. Internet of things 2. Blockchain Technology 3. Cloud technology. The internet of things is used for asset tracking and available stock tracking in the shop. This asset tracking on the movement of truck information is updated in the blockchain in real-time. This asset tracking information is available and can be accessed by anyone by locking in the blockchain. The system allows moving all the transactions in blockchain by the smart contract-based transaction. The proposed system design consists of the following block number 1 . Service Kiosk with the user interface 2. Backend blockchain network 3. Communication stack module for movement of the transaction and data access from local system to blockchain network 4. Google speech engine system for local language voice interaction. Fig. 1 provides the overall framework of the proposed system design

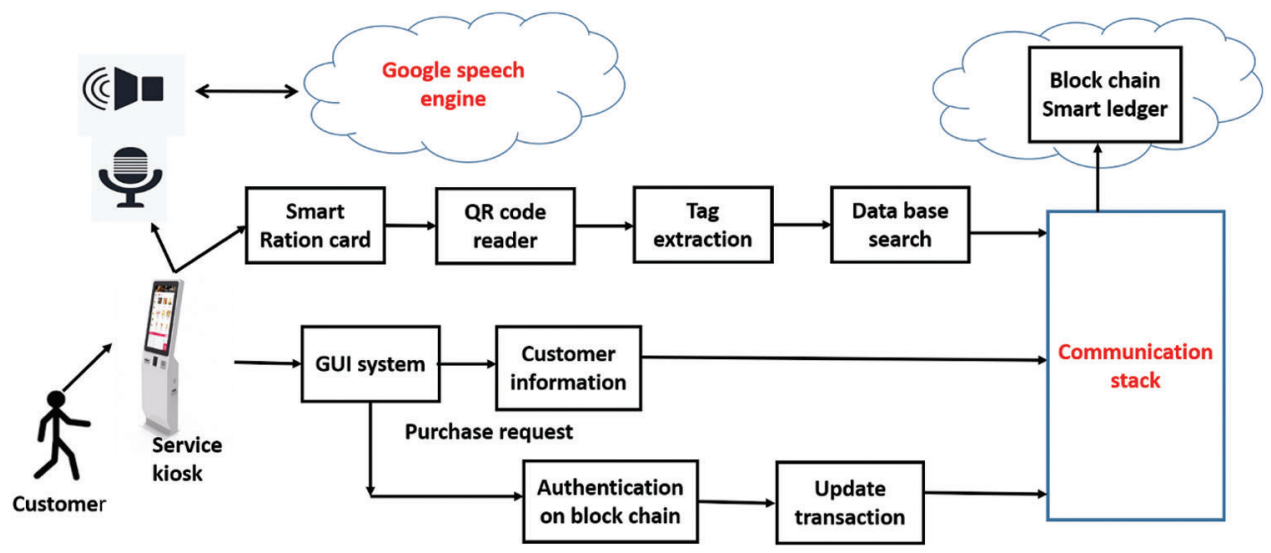

Figure 1: Framework of blockchain-based smart ration shop system

The Embedded hardware processor raspberry-pi is used for implementing the system. An embedded Raspberry-pi-based peer nodes are deployed for blockchain implementation. For a user-friendly kiosk system Raspberry pi based embedded system is deployed to provide Tamil native language GUI interaction and voice-based interaction with the system.

\subsection{The Service Kiosk}

The Service Kiosk has subsystems for the following purpose :

- user interface model to provide customers interaction in Tamil using Text and Voice interface

- input module, which is using Aadhar card QR Code scanner which will act as a key to access the database of ration shop and do the transaction

- An output user interface to Displays required information to users like the quantity of goods bought and the amount left in the storage.

- The proposed system provides dynamic webpage with native Tamil language and attractive GUI (Graphical User Interface) to access the database of entire local ration shop as well as the entire country ration shop stocks. This will provide more transparency of stock availability with live updates and overcome the problem of black market selling. But the existing SMS system only provides how much goods are purchased for the particular user, not the local and global database access.

\subsection{Blockchain Network Module}

The blockchain network is designed to ensure the following aspect in the proposal smart ration shop. 
- To Ensures Immutability of records

- To avoid Local vendors taking advantage of rural people by Providing wrong stack information through the storage of stock information and transparent data access in the blockchain network.

- Avoiding manipulated price hikes as the prices will be set by the government and available in the blockchain network for any time access.

- Providing direct linkage and communication to the service kiosk so that real-time updates will be happening

- Providing full authority to government agencies using a private blockchain so that the central government has total control over the database

- Using hyperledger, so that the central government can keep updating the consensus and keep a record of the database

This study implements blockchain technology using a private ethereum network. One of the requirement of implementing a blockchain network is it has to run on multiple nodes. The blockchain component is essentially a Private Ethereum network running on Raspberry Pis, acting as the multiple nodes in this study. Each of the nodes has a complete copy of the chain, and to append any data, which has to be verified by at least $51 \%$ of the nodes. The data already in the chain cannot be modified, and this means there's no room for any manipulation by any bad actors in the complete system.

Our network uses an alternative Consensus mechanism, 'Proof of Authority (PoA), rather than the default mechanism, 'Proof of Work'(PoW). This is for several reasons, as mentioned below:

- Owing to the huge computational load that the default consensus mechanism PoW requires, it is not viable to have a PoW network running on Raspberry Pis.

- A PoW network owing to an increase in computation power, will use more energy, which is not ideal.

- PoA allows us only to choose the nodes which will be making the blocks, thus allowing us to keep the network restricted to verified nodes.

The network can be expanded by adding more nodes to the network since the more nodes, the harder the network is to attack. When a new node joins the network, it searches for a nearby boot node, which sends the data about the other nodes. Hence the new node can connect to the other nodes already in the network and start syncing the chains. The new node once has the data till the head block; it is entirely set up and ready to mine. The whole process is automatic. Apart from the actual network, another critical aspect is the smart contract running on the network, which is where all the computing takes place. Web3 API is used to connect the blockchain to the frontend. It allows us to use the functions specified in the smart contracts.

Fig. 2 shows the integration of the blockchain network with the smart ration shop system, where three blockchain nodes are implemented in a single raspberry pi module, which is communicating to the frontend user interface through a backed script. Since the Indian citizen author card is accepted as an authority card, which is utilized as an access key for accessing the blockchain network. Here, the back-end script is interacting with the QR scanner, which is scanning the QR code available in the smart ration card of the user. The QR scanner Provides information from the aadhar card to use it as an access key to the blockchain network.

\subsection{QR Scanning Implementation for Asset Tracking and Ration Card User Information Reading}

Asset tracking and Ration Card QR Code Scanning both will be taken care of by a simple set up of the QR code scanner. Under asset tracking, the sacks containing various grains and pulses (or other goods) will be scanned with the help of QR code at every checkpoint. Hence, with the integration of Blockchain, a foolproof system will be established. At the ration shop side, the users can scan their ration cards at the available 
kiosk systems. Hence, we will be able to monitor the flow of goods to each customer. QR code Scanner setup is having mainly a camera connected to a controller.

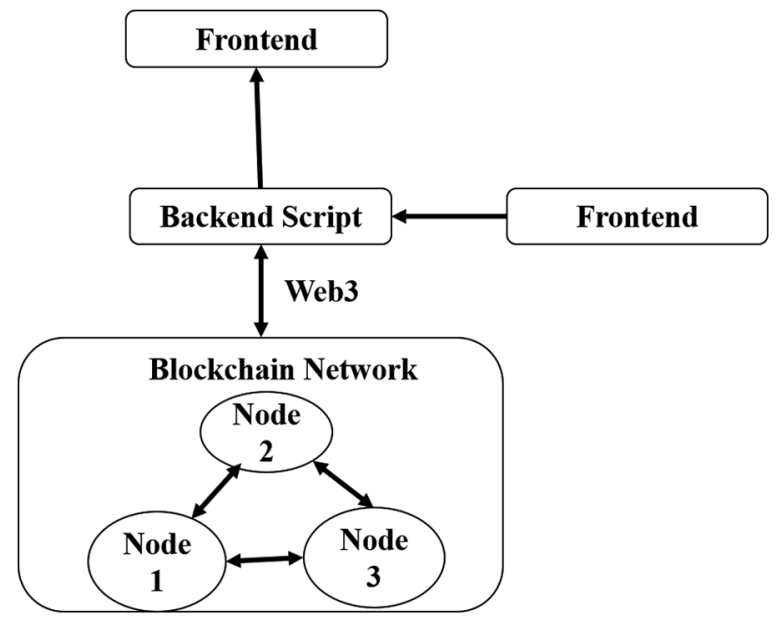

Figure 2: Blockchain integration in the smart ration shop system

Our setup of the QR code scanner involves a Raspberry Pi and a Pi Cam. For this work, we installed the zbarlight library. We kept things simpler by avoiding the use of OpenCV. The zbarlight library acts as the brain of the entire setup. It is because it decodes the image of the QR code into necessary codes.

Once the QR image is captured from the camera, the system will check if the captured picture has the QR code and validity of the code. If the QR code is not found or the code is invalid, a user voice command and text information will be provided to the user to give proper input. Once a valid QR code is received from the module, then, using the HTTP method of REST post commands, this code information is transferred to the backend script via the proper IP address.

\subsection{Software Stack}

The Software stack of the smart Public Distribution System consists of a back-end module running on NodeJS, Front-End Interfaces, which are implemented as WebApps, and REST API based communication modules for communication between the various components over HTTP. The NodeJS scripts further use Linux Bash Scripts to interface with the Raspbian Operating System of Raspberry Pi hardware for tasks such as playing audio. The Web3 API is used to communicate with the Ethereum smart contracts. The QR code present on the ration card is used as a unique identifier. Since the QR Code only contains a hash and not the User Details, the same is retrieved by cross-referencing the hash with a database. This hash value is used to fetch the quota details of the user from the blockchain and allow him to make a purchase. Once the user has chosen the Item and the quantity, a text-to-speech script narrates the option in the local language. Upon confirmation, the transaction is recorded in the blockchain.

Fig. 3 shows the overall interaction of the software modules in the smart ration shop system.

The algorithm for the software stack is represented below

step1: Run frontend GUI routine

a) Get user smart card detail from QR scanner

b) Validate received details format

c) call backend script 
step 2: Run backend script

a) Search in the database for the entry of the user

b) Populate user stock details

c) Call frontend to interact with the purchase transaction and get transaction detail

d) Call blockchain module to register the transaction in the blockchain network

1) Broadcast transaction

2) validate transaction

3) Add to blockchain

e) call frontend to show the success of the transaction to the user

Blockchain algorithm:

step 1: Employ the user transaction data to generate HASH value.

step 2: Apply the consensus algorithm, Call Proof of Authority (PoA) algorithm

step 3: Create block add to the blockchain

Algorithmic supporting format relates to blockchain FPS is given below

\section{Block creation using Proof of Authority}

The new block is created using leader node, which is defined as follows:

1. Gather updated transactions from its own transaction queue.

2. Run the transactions by line. Invalid Transactions are rejected or not executed.

3. Check the error for block creation limits.

4. Generate one block with respect to valid transactions and indicate it by its node's private key (ECDSA algorithm).

5. Forward that block to another validating node.

\section{New block validation using Proof of Authority}

Validating other nodes:

1. Accept the latest/new block and validate that:

- The leader node creates a new block with the current interval.

- None of the other blocks are generated at the current interval by the leader node.

- Finally, the block is generated and marked correctly.

2. Run the transaction block in line. Examine that some transactions are executed within limits of generated blocks successfully.

3. Reject or Add the block with respect to the previous step:

- Once block validation is fruitful, attach the updated block to the blockchain nodes.

- For failed block validation, Block will be rejected and forward it as a bad transaction block. If the invalid block generation is continued by any node, it will be blocked and removed from the valid node list.

\section{Deployment and Experimental Results}

The proposed system is deployed in the Anju village near Chennai for evolution and benchmarking. After deployment, the Ease of usability among the villagers on different groups of people like genderwise, educational background-wise, and age-wise is analyzed. Under this usability test, the following 
aspects are taken into account and evaluated the performance. 1. Ease of learning 2. Efficiency of use 3. Memorability 4. Error frequency and severity 5. Subjective satisfaction. The evaluation and results are tabulated in the Tab. 1 Under the four categories given in the table, 20 people, are used per category for evolution and in each group that ten male candidates and ten female candidates are used. After deploying the system, the system is given to the user to use a system for ten days based on that experience on ten days usage; the users are then given the survey form to collect the data required for the above analysis. We can see that the educated background People have good satisfaction values in all the categories comparing to the uneducated background. Anyhow, the number in Tab. 2 shows that the proposed system can provide a good experience of users and make the user use the system in the most comfortable best way, efficient way. In the gender category_ wise analysis, uneducated Female people perform very well and can use the product officially comparing illiterate male people. Undereducated categories of people, both female and male, perform mostly in a similar way. Age-wise if you see, mostly the people with less than 40 age providing satisfaction value comparing 50 and above.

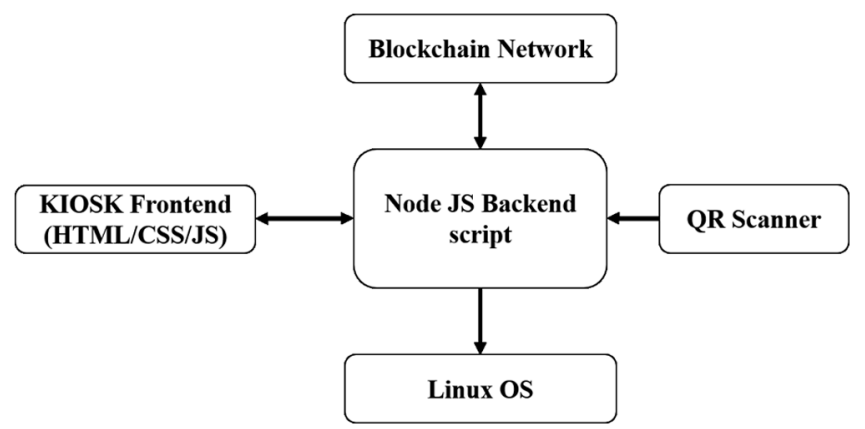

Figure 3: Software stack of smart ration shop

Here, the efficiency term indicates how quickly a user can use the system after several usages of the proposed system. Thus from the above result, we can conclude that the proposed system can provide Good usability and satisfaction, which ensures that confidence deploys the system for the village people.

The computational complexity of the proposed blockchain system is also tested and evaluated in terms of the average number of transactions that can be generated by the system by varying the number of transaction requests per second. A random arrival of transactions is generated for that the number of successful transactions that can be provided by the system Is recorded and plotted in Fig. 4.

Fig. 4 shows that the proportion of Successful transactions decreases with respect to the number of transaction arrivals. It can also be observed that after 40 transaction arrival rates, the portion of a successful transaction is constant, and it continues for any arrival rate. This is happening because, in the Blockchain network, the block size is fixed, so we cannot add more than some number of transactions in the given block.

The time delay of generating a new transaction is evaluated by injecting five transactions, which is reported in the Tab. 2.

Tab. 3 results show that the delay for generating new blocks varies from 160 up to 250 milliseconds for the case of the POW mining algorithm. For the POA algorithm, the time delay ranges from 100 to 130 based on the network traffic at the time.

The traffic handling capacity of the blockchain is also evaluated in terms of delay by varying the number of nodes participating in the network. This time-day analysis is provided in Fig. 5. The robustness of the proposed system is tested by admitting a number of fake transactions on a given amount of 100 total transactions. Here, a variety of fake transactions are injected into a hundred transactions, and the percentage of admitted transactions by the blockchain network is analyzed with respect to these fake transactions. 
Fig. 5 shows the normalized delay values for the given number of nodes, which is increasing linearly.

30 percent to 90 of percentage correct transactions in the blockchain is observed, when the varying number of fake transactions is injected. The results in Fig. 6 clearly show that when the number of fake transactions increases, then the percentage of the correct transaction is decreased. This graph proves that the blockchain network can reject fake transactions, which proves the robustness of the proposed system.

Table 2: Usability analysis results

\begin{tabular}{|c|c|c|c|c|c|c|c|c|c|c|}
\hline \multirow[t]{2}{*}{ Category of people } & \multicolumn{2}{|c|}{$\begin{array}{l}\text { Ease of } \\
\text { learning } \\
\text { In } \%\end{array}$} & \multicolumn{2}{|c|}{$\begin{array}{l}\text { Efficiency of } \\
\text { use In } \%\end{array}$} & \multicolumn{2}{|c|}{$\begin{array}{l}\text { Memorab- } \\
\text { ility } \\
\text { In \% }\end{array}$} & \multicolumn{2}{|c|}{$\begin{array}{c}\text { Error } \\
\text { frequency } \\
\text { and severity } \\
\text { In } \% \\
\end{array}$} & \multicolumn{2}{|c|}{$\begin{array}{l}\text { Subjective } \\
\text { satisfaction } \\
\text { In } \%\end{array}$} \\
\hline & Male & Female & Male & Female & Male & Female & Male & Female & Male & Female \\
\hline $\begin{array}{l}\text { Age } 25 \text { with good } \\
\text { education background }\end{array}$ & $98 \%$ & $99 \%$ & $100 \%$ & $100 \%$ & $99 \%$ & $99 \%$ & $0 \%$ & $0 \%$ & $100 \%$ & $100 \%$ \\
\hline $\begin{array}{l}\text { Age } 25 \text { without } \\
\text { education background }\end{array}$ & $89 \%$ & $91 \%$ & $99 \%$ & $99 \%$ & $98 \%$ & $99 \%$ & $1 \%$ & $1 \%$ & $100 \%$ & $100 \%$ \\
\hline $\begin{array}{l}\text { Age } 35 \\
\text { with good education } \\
\text { background }\end{array}$ & $95 \%$ & $97 \%$ & $98 \%$ & $99 \%$ & $97 \%$ & $97 \%$ & $2 \%$ & $3 \%$ & $99 \%$ & $100 \%$ \\
\hline $\begin{array}{l}\text { Age } 35 \\
\text { without education } \\
\text { background }\end{array}$ & $91 \%$ & $93 \%$ & $92 \%$ & $92 \%$ & $94 \%$ & $3 \%$ & $1 \%$ & $93 \%$ & $98 \%$ & $99 \%$ \\
\hline Age $>50$ with education & $84 \%$ & $80 \%$ & $85 \%$ & $84 \%$ & $90 \%$ & $4 \%$ & $4 \%$ & $80 \%$ & $91 \%$ & $91 \%$ \\
\hline $\begin{array}{l}\text { Age }>50 \text { without } \\
\text { education background }\end{array}$ & $79 \%$ & $77 \%$ & $80 \%$ & $80 \%$ & $80 \%$ & $4 \%$ & $4 \%$ & $77 \%$ & $90 \%$ & $89 \%$ \\
\hline
\end{tabular}

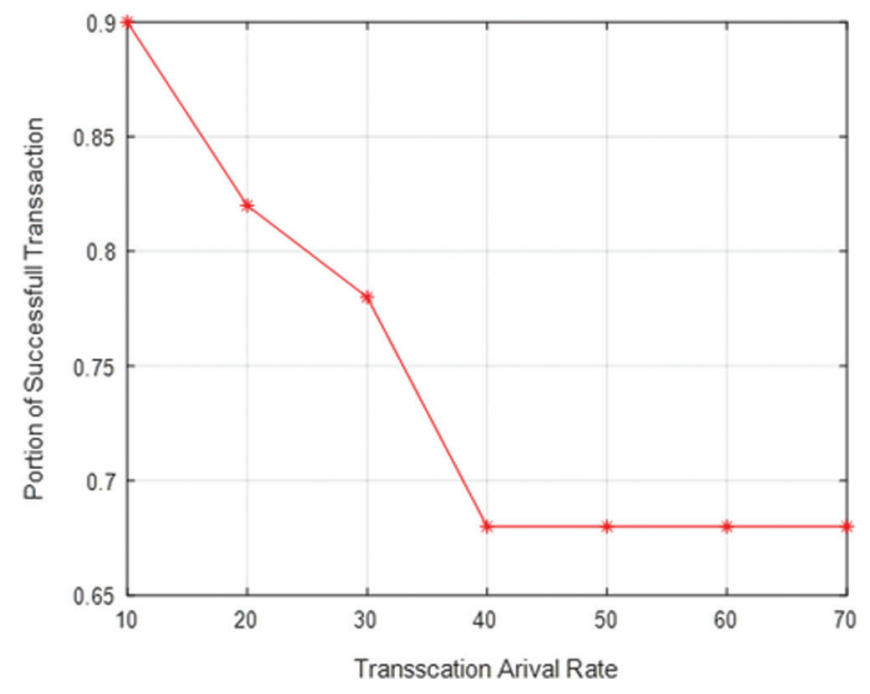

Figure 4: Success full traction rate with respect to the number of transaction arrival 
IASC, 2022, vol.32, no.1

Table 3: Time delay for generating a new block

\begin{tabular}{lll}
\hline Transaction & Time in ms for POW & Time in ms for POA \\
\hline 1 & 250 & 130 \\
2 & 180 & 121 \\
3 & 178 & 118 \\
4 & 160 & 100 \\
5 & 162 & 108 \\
\hline
\end{tabular}

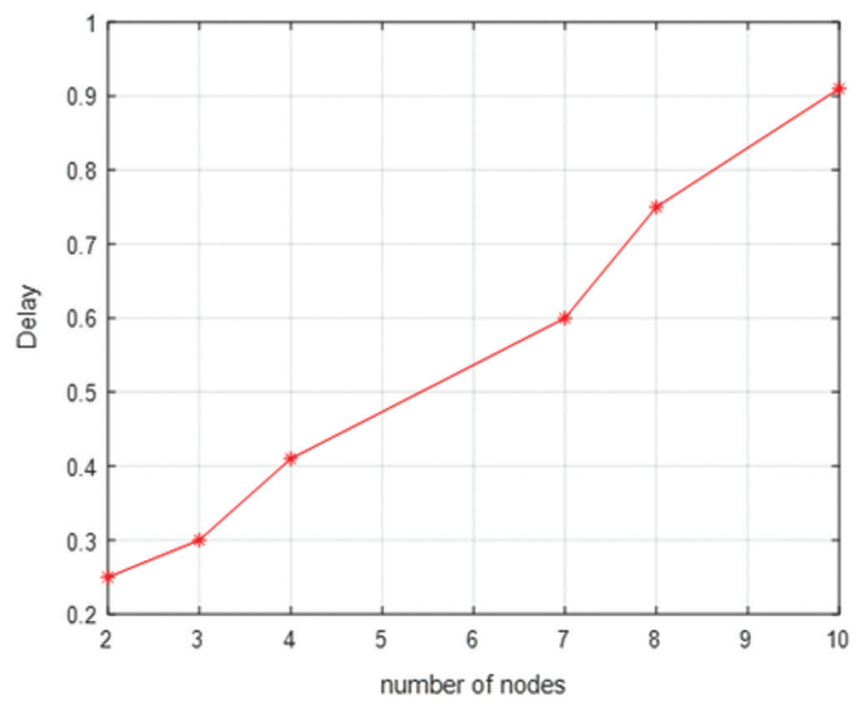

Figure 5: Delay with respect to the number of nodes

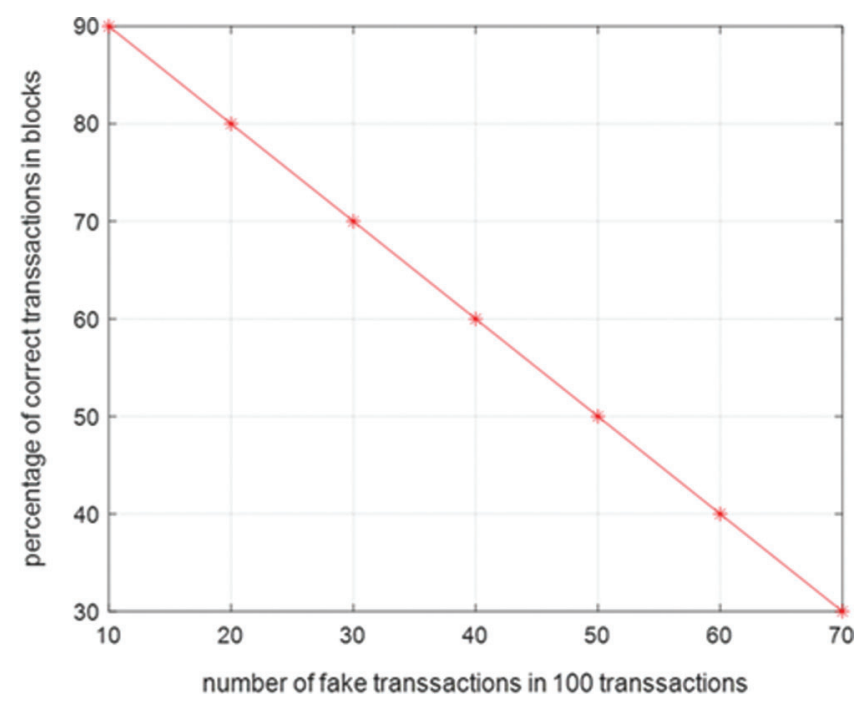

Figure 6: Fake transaction admission rejection analysis 


\section{Conclusion}

The work provides a secure transaction and data management to avoid the frequent exploitation faced by villagers. The proposed system ensures that this system can be used easily and efficiently by every individual in the village, and they can receive their provisional at a fair price with full transparency. The effectiveness of voice interactive kiosk systems is measured subjectively, and the satisfaction levels are reported as $90 \%$ on average. Even illiterate villagers will be able to access their fair shop transaction details with a user-friendly voice interaction engine. They can talk with their mother language Tamil with the system. The immutability of the transaction is measured, which proves that the system is $100 \%$ immutable. The response time of the system is around 130 milliseconds, which makes the system perfect for real-time operation. The system will enable asset tracking while transporting the fair shop goods from distribution points to the shop using QR codes and checkpoint places. This kind of asset tracking enables transparency on products' transports and avoids theft and black market selling of the items.

Acknowledgement: This work was supported by the Unnat Bharat Abhiyan (UBA) - A flagship program of the Ministry of Education, Government of India.

Funding Statement: The authors received no specific funding for this study.

Conflicts of Interest: The authors declare that they have no conflicts of interest to report regarding the present study.

\section{References}

[1] A. N. Shwetha and C. P. Prabodh, "Blockchain - bringing accountability in the public distribution system," in 4th Int. Conf. on Recent Trends on Electronics, Information, Communication \& Technology (RTEICT), Bangalore, India. pp. 330-335, 2019.

[2] A. P. B. S. S. Shukla, "A step towards smart ration card system using RFID \& IoT," in Int. Conf. on Smart City and Emerging Technology (ICSCET), Mumbai, India. pp. 1-5, 2018.

[3] L. Lao, Z. Li, S. Hou, B. I. N. Xiao and T. Hong et al. "A survey of IoT applications in blockchain systems : Architecture, consensus, and traffic modeling," ACM Computing Surveys, vol. 53, no. 1, pp. 1-32, 2020.

[4] P. Cui, J. Dixon, U. Guin and D. Dimase, "A blockchain-based framework for supply chain provenance," IEEE Access, vol. 7, pp. 157113-157125, 2019.

[5] E. Kaur and A. Oza, "Blockchain-based multi-organization taxonomy for smart cities," SN Applied. Sciences., vol. 2, no. 3, pp. 1-11, 2020.

[6] Y. P. Tsang, K. L. Choy, C. H. Wu, G. T. S. Ho and H. Y. Lam, "Blockchain-driven iot for food traceability with an integrated consensus mechanism," IEEE Access, vol. 7, pp. 129000-129017, 2019.

[7] Y. Wang, "Designing a blockchain enabled supply chain," International Federation of Automatic Control Papers, vol. 52, no. 13, pp. 6-11, 2019.

[8] H. J. Scholl, "Digital government: Looking back and ahead on a fascinating domain of research and practice," Digital. Government. Research. Practice, vol. 1, no. 1, pp. 1-12, 2020.

[9] F. M. Benčić, P. Skočir and I. P. Zarko, "DL-tags: DLT and smart tags for decentralized, privacy-preserving, and verifiable supply chain management," IEEE Access, vol. 7, pp. 46198-46209, 2020.

[10] X. Xu, F. Rahman, B. Shakya, A. Vassilev and D. Forte et al., "Electronics supply chain integrity enabled by blockchain," ACM Transactions on Design Automation of Electronic Systems, vol. 24, no. 3, pp. 1-25, 2019.

[11] Y. Li, "Emerging blockchain-based applications and techniques," Service Oriented Computing and Applications, vol. 13, no. 4, pp. 279-285, 2019.

[12] G. Ishmaev, "The ethical limits of blockchain-enabled markets for private IoT data," Philosophy. Technology, vol. 33, no. 3, pp. 411-432, 2019. 
[13] Q. Lin, H. Wang, X. Pei and J. Wang, "Food safety traceability system based on blockchain and EPCIS," IEEE Access, vol. 7, pp. 20698-20707, 2019.

[14] L. He, M. Xue and B. Gu, "Internet-of-things enabled supply chain planning and coordination with big data services: Certain theoretic implications," Journal of Management Science and Engineering, vol. 5, no. 1, pp. $1-22,2020$.

[15] O. Poenicke, M. Groneberg and K. Richter, "Method for the planning of IoT use cases in smart logistics zones," International Federation of Automatic Control Papers, vol. 52, no. 13, pp. 2449-2454, 2019.

[16] C. Marinagi, P. Belsis and C. Skourlas, "New directions for pervasive computing in logistics," Procedia-Social and Behavioral. Sciences., vol. 73, pp. 495-502, 2013.

[17] J. L. Zhao, S. Fan and J. Yan, "Overview of business innovations and research opportunities in blockchain and introduction to the special issue," Financial. Innovation., vol. 28, no. 2, pp. 1-7, 2016.

[18] G. Prause, "Smart contracts for smart supply chains," IFAC-PapersOnLine, vol. 52, no. 13, pp. 2501-2506, 2019.

[19] Y. Issaoui, A. Khiat, A. Bahnasse and H. Ouajji, "Smart logistics: Study of the application of blockchain technology," Procedia Computer. Science, vol. 160, pp. 266-271, 2019.

[20] S. Wang, D. Li, Y. Zhang and J. Chen, "Smart contract-based product traceability system in the supply chain scenario," IEEE Access, vol. 7, pp. 115122-115133, 2019.

[21] Y. Kayikci, "Sustainability impact of digitization in logistics," Procedia Manufuters, vol. 21, pp. 782-789, 2018.

[22] A. F. Famnaz and S. Kumari, "Implementing blockchain in public distribution system," International Journal of Advanced Research in Computer and Communication Engineering, vol. 8, no. 6, pp. 120-125, 2019.

[23] S. Dhanake, S. Desale, P. Pawar, G. Patil and S. Shende, "Blockchain technology in public ration distribution," International Research Journal of Engineering and Technology (IRJET) vol. 8, no. 3, pp. 732-736, 2021.

[24] P. Thakare, N. Dighore, A. Chopkar, A. Chauhan and D. Bhagat et al., "Implementation of block chain technology in public distribution system," in Int. Conf. on Hybrid Intelligent Systems, Springer Cham., pp. 210-219, 2021.

[25] V. Ponnusamy, R. Nandakumar, P. Gouthaman, S. Yuvaraj and C. Clement et al. "A review on blockchain technology applications with an emphasis on health care," International Journal of Advanced Science and Technology, vol. 29, no. 7s, pp. 4835-4839, 2020. 